\title{
Pulmonary nocardiosis in a patient with pemphigus foliaceus: case report and literature review
}

\author{
Ning Luo ${ }^{1 \dagger}$, Shifan Tan ${ }^{1 \dagger}$, Xiaocai Li ${ }^{1}$, Si Liu', Shivank Singh², Mafeng Chen ${ }^{3}$, Weiye Yang ${ }^{4}$, Yanhong He ${ }^{5}$, \\ Chunna Chen ${ }^{6}$ and Min Liang ${ }^{1 *}$
}

\begin{abstract}
Background: Nocardiosis is an uncommon opportunistic infection seen in immunocompromised patients or those with a dysfunctional immune system. Nocardia asteroides infection in patients with Pemphigus foliaceus (PF) has never been reported.

Case presentation: We report an interesting case of nocardiosis-characterized by pulmonary intra-cavitary infection, in a 54-year-old man with PF and diabetes mellitus. The man finally recovered from the infection.

Conclusions: This is the first case reporting pulmonary nocardiosis in a patient with PF. We recommend that physicians be aware of nocardiosis in patients with pemphigus as a possible cause of underlying infectious disease to avoid misdiagnoses and mismanagement.
\end{abstract}

Keywords: Nocardia asteroides, Pemphigus foliaceus, Diabetes, Corticosteroids, Case report

\section{Background}

Pemphigus is a chronic, potentially lethal disorder of the skin and mucous membranes. Long-term therapy with corticosteroids to control disease might, unfortunately, cause generalized immuno-suppression. We present a rare case, in which immuno-supression was further potentiated by diabetes mellitus, resulting in an opportunistic pulmonary infection with Nocardia asteroides.

\section{Case presentation}

This case report has been approved by the ethics committee of Maoming People's Hospital.

A 54-year-old man with diabetes mellitus was admitted to Maoming People's Hospital on 26, April 2020. He

\footnotetext{
* Correspondence: imtuaska@163.com

${ }^{\dagger}$ Ning Luo and Shifan Tan contributed equally to this work.

'Department of Respiratory and Critical Care Medicine, Maoming People's Hospital, MaoNanQu, WeiMing Road 101 Hao, Maoming City, Guangdong, China

Full list of author information is available at the end of the article
}

was diagnosed with PF ten months prior to admission. The diagnosis was based on clinical manifestations and laboratory findings [1], 1) Localized superficial blisters with erosion and crusts appeared on the area of upper trunk (Fig. 1.) 2) Punch biopsy from anterior chest revealed decreased intercellular adhesions and acantholysis (Fig. 2.) 3) Direct immunofluorescence showed intercellular IgG deposition in the epidermis (Fig. 3.) 4) Detection of serum anti-desmoglein-1 (Dsg-1) antibody with a level more than $150 \mathrm{U} / \mathrm{mL}$ (ELISA method) whereas the serum anti-desmoglein-3 (Dsg-3) antibody was within the normal range (Fig. 4.).He was initially prescribed daily oral prednisone $(60 \mathrm{mg})$ for PF and achieved complete remission. After 3 months of maintenance therapy, the dose of prednisone was gradually and carefully tapered to $20 \mathrm{mg}$ P.O. daily. The patient was also diagnosed with diabetes mellitus 1 year ago, but did not follow medical advice for management. Consequentially, he had poorly-controlled blood glucose levels. He

C C The Author(s). 2020 Open Access This article is licensed under a Creative Commons Attribution 4.0 International License, which permits use, sharing, adaptation, distribution and reproduction in any medium or format, as long as you give appropriate credit to the original author(s) and the source, provide a link to the Creative Commons licence, and indicate if changes were made. The images or other third party material in this article are included in the article's Creative Commons licence, unless indicated otherwise in a credit line to the material. If material is not included in the article's Creative Commons licence and your intended use is not permitted by statutory regulation or exceeds the permitted use, you will need to obtain permission directly from the copyright holder. To view a copy of this licence, visit http://creativecommons.org/licenses/by/4.0/ The Creative Commons Public Domain Dedication waiver (http://creativecommons.org/publicdomain/zero/1.0/) applies to the data made available in this article, unless otherwise stated in a credit line to the data. 


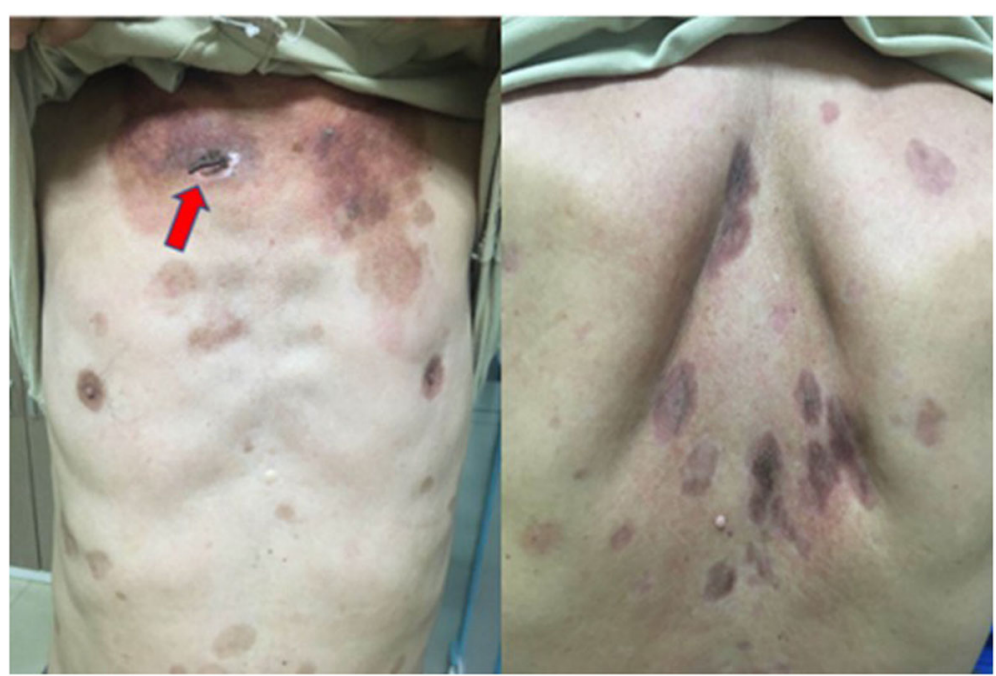

Fig. 1 Multiple, crusted lesions of PF on the anterior chest and upper back (the scar is marked with a red arrow)

worked as a farmer and had a 10 pack-year smoking history.

Three months before admission the patient was wounded by a tree branch on the anterior chest but didn't seek medical service at the time. He began feeling fatigued about a month later which was followed gradually by a productive cough and right-sided chest pain. The patient presented to the local clinic with a sudden high grade fever $\left(39.6^{\circ} \mathrm{C}\right)$, chills, sputum evolution and shortness of breath, where he was thought to have bacterial pneumonia and received oral and intravenous antibiotic therapy. However, he failed to respond to the therapy and was transferred to Maoming People's Hospital.

During primary evaluation, the patient exhibited a poor general condition and strong breathlessness. Initial

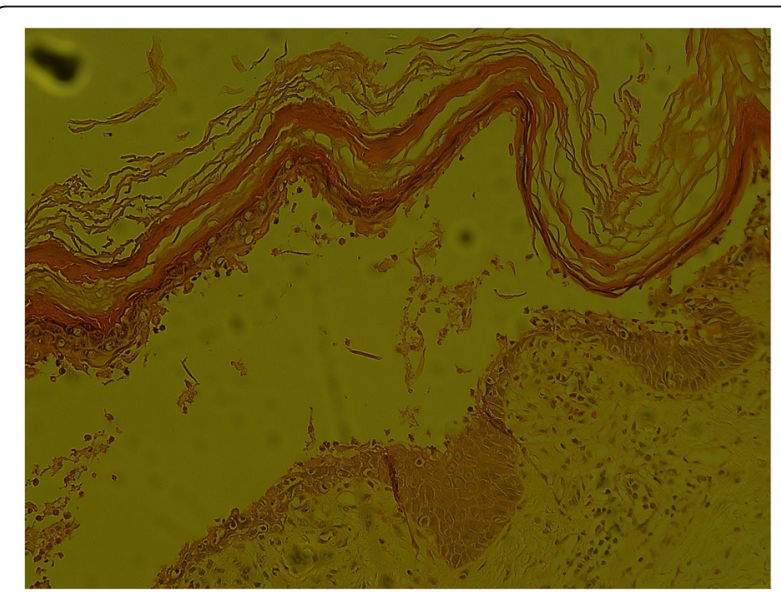

Fig. 2 Punch biopsy from the anterior chest revealed decreased intercellular adhesions and acantholysis (Hematoxylin \& Eosin, $\times 200$ ) vital signs were: Body temperature: $39.4{ }^{\circ} \mathrm{C}$; Heart rate: $116 \mathrm{bpm}$; Respiratory rate: 26 breaths/min; Blood pressure: 101/60; Oxygen saturation: 75\%. Physical examination revealed multiple skin lesions on the chest, shoulder, and upper back. An old scar was visible on the right sternal border- on the same area as the skin lesions (Fig. 1.). Lung auscultation revealed end-inspiratory rales in both lung bases. Blood tests showed a WBC count of 12,910 cells/ul, Rapid C-Reactive Protein count of over $200 \mathrm{mg} / \mathrm{L}$ and Hemoglobin count of $11.7 \mathrm{~g} / \mathrm{dl}$. His glycated hemoglobin (HbA1c) was $10.10 \%$.Human immunodeficiency serology was negative. A chest CT was performed and revealed several 'fungal ball' like inclusions in thick-walled cavities present in multiple lobes of both lungs (Fig. 5.).Blood culture, Tuberculin test, Xpert MTB/RIF test, serum $(1,3)-\beta$-D-glucan (BDG) and serum

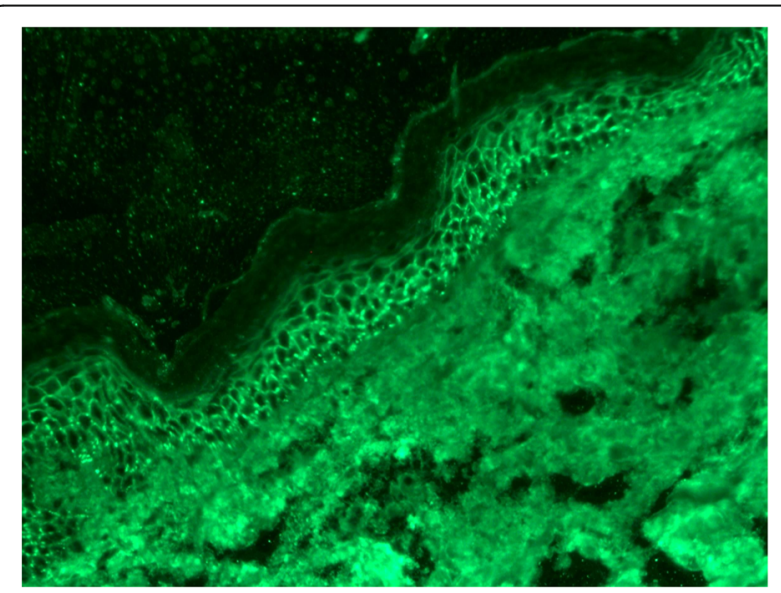

Fig. 3 Direct immunofluorescence: Intercellular immune lgG deposits present in the epidermis 


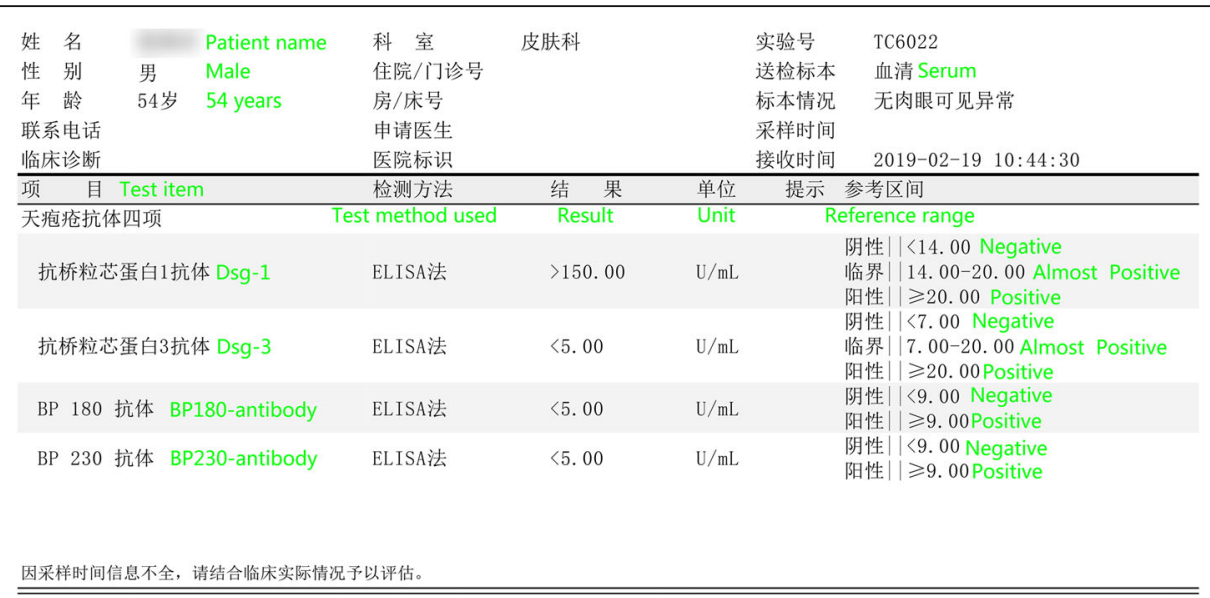

Fig. 4 Results of serological findings (ELISA method). Dsg-1 antibody with a level more than $150 \mathrm{U} / \mathrm{mL}$, Dsg-3 antibody was within the normal range
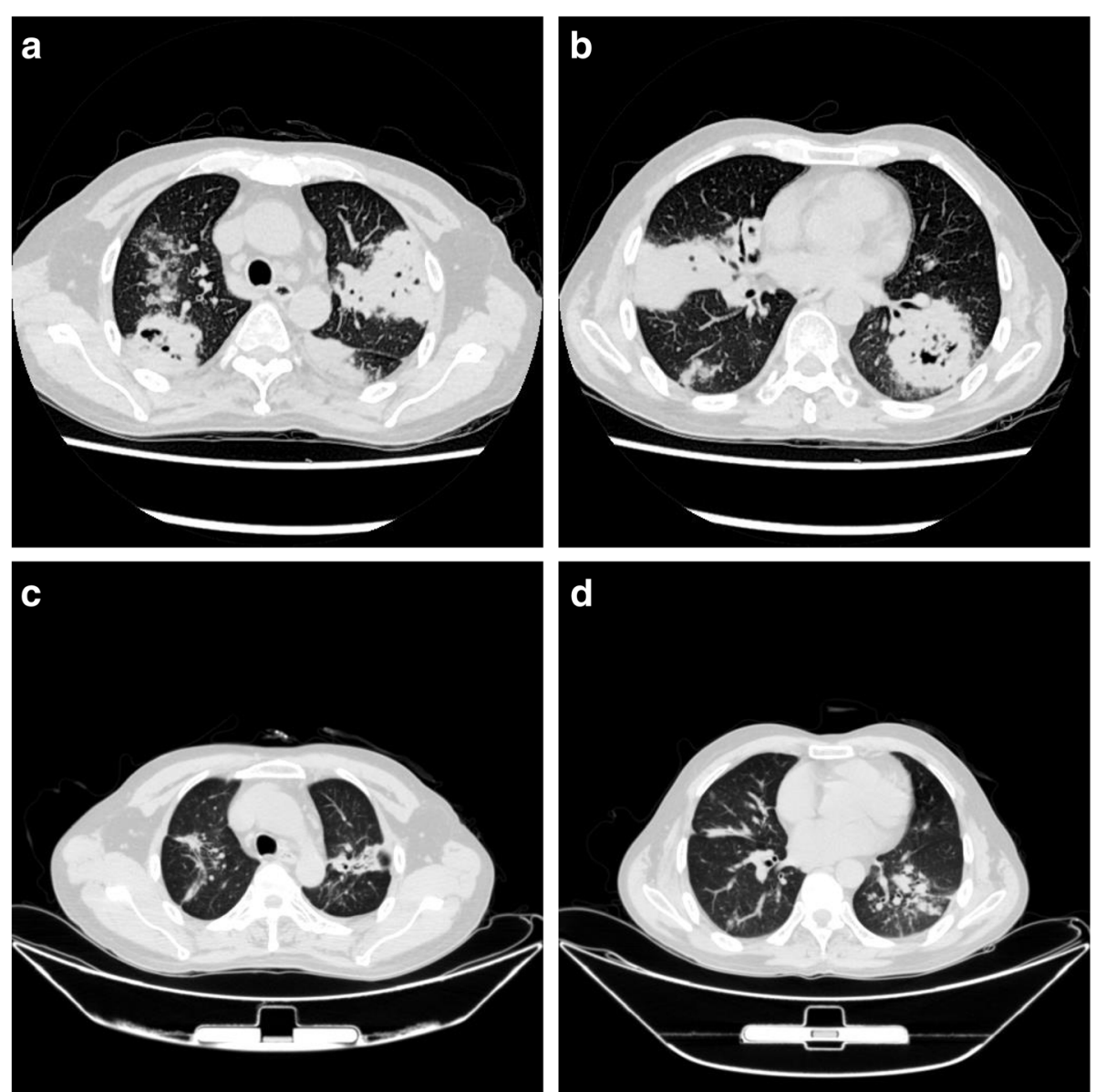

Fig. 5 CT comparison at hospital admission ( $\mathbf{a}$ and $\mathbf{b}$ ) and 3 months post hopital-discharge (c and $\mathbf{d}$ ); On admission: Thick-walled cavities in multiple lobes of both lungs (a: upper lobes, $\mathbf{b}$ : Middle lobe and lower lobes); $\mathbf{c}$ and $\mathbf{d}$ : lesions improved following treatment for 3 month 


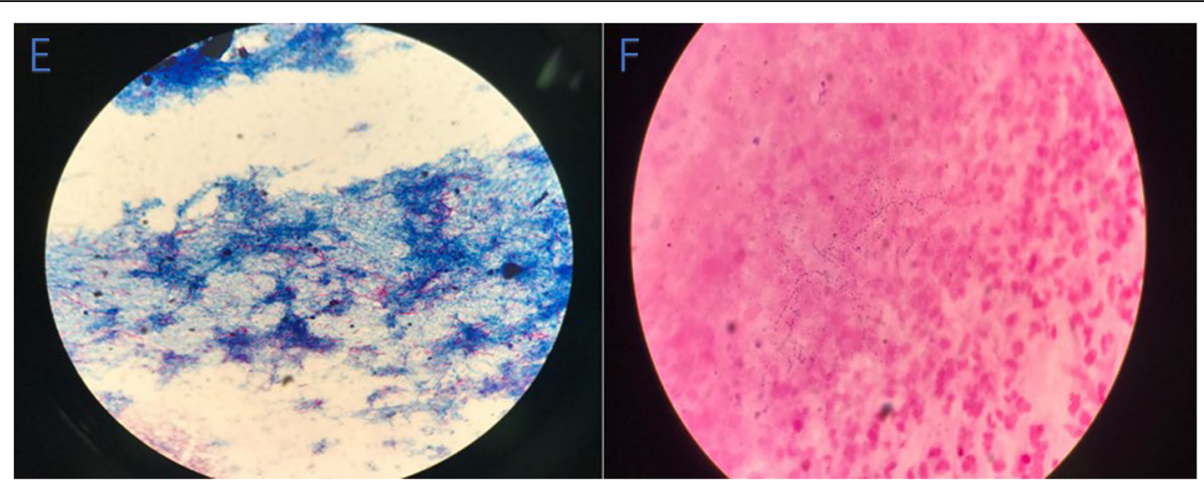

Fig. 6 Results of sputum culture. E: Branching, thin filamentous acid-fast bacteria on modified (1\%) ZN stain, F: Gram-positive, filamentous, delicate branching rods with an irregular staining pattern

galactomannan (GM) antigen detection test returned negative. However, specimen from Broncho-alveolar Lavage fluid (BAL) and multiple sputum cultures revealed Nocardia spp. (Fig. 6.). The organism was later identified as Nocardia brasiliensis by rRNA gene sequencing. Results of other complementary studies to detect extra-pulmonary organ involvement were negative.

A combination of intravenous cefotaxime (2.25 g,Q8h), amikacin $(0.4 \mathrm{~g} /$ day) and oral trimethoprim-sulfamethoxazole $(0.32 \mathrm{~g}$ trimethoprim, $1.6 \mathrm{~g}$ sulfamethoxazole) daily were initiated after diagnosing Nocardia asteroides, along with continuation of prednisone $(20 \mathrm{mg} /$ day $)$ for pemphigus. Insulin was also administered to manage blood glucose levels.

Seven days post-admission, his body temperature became normal and we was told by the patient that the symptoms of cough and breathlessness were markedly improved as well. Blood tests showed WBC counts and levels of Rapid C-Reactive protein within the normal range. Following another 7 days of sequential therapy, the patient was discharged with instructions to continue therapy with oral trimethoprim-sulfamethoxazole for next 3 months.

After discharge, the patient did well. His pemphigus was completely under control with a daily oral dose of $20 \mathrm{mg}$ prednisone and progressive improvements took place in cough and breathlessness up to the last visit on August 1, 2020. A follow-up CT was performed in the meanwhile, which revealed further improvement from nocardia pneumonia (Fig. 5.).

\section{Discussion and conclusion}

Pemphigus is a rare but serious chronic blistering disorder of the skin and mucous membranes [2]. It is usually categorized into two distinct forms, Pemphigus vulgaris (PV) and Pemphigus foliaceus (PF), each with its own variants(e.g. The endemic form of PF, also known as Fogo selvagem (FS)) [3-5]. Over the past decades, rare forms of pemphigus have been described, including paraneoplastic pemphigus, IgA pemphigus, and pemphigus herpetiformis, etc. [5]. Even though the disease has a chronic clinical course, the risk of morbidity and mortality is considerable. Before the advent of corticosteroids, estimated mortality rate at the end of 5 years was approximately 100\% [6]. With the availability of corticosteroids, the mortality rate has declined to 5-10\% [7].

Bacterial infection with Staphylococcus aureus, Proteus vulgaris and Pseudomonas aeruginosa, is the most common complication after long-term administration of corticosteroids in pemphigus patients. However, pulmonary nocardiosis in a patient with pemphigus has been rarely reported in recent cases.

Pulmonary nocardiosis is an opportunistic infection, which usually occurs in immunocompromised patients, alcoholics, and patients on long term immunosuppressive therapy [8]. It has a variable presentation on Chest CT, usually mimicking tuberculosis, fungal balls, or lung cancer [9]. Isolation and identification of Nocardia spp. from cultures of sputum, BAL, and biopsy helps establish the diagnosis [10]. A combination of sulfonamides, amikacin, and ceftriaxone is recommended as first-line therapy [11]. In our patient's case, the long-term management of pemphigus with oral prednisone and poor diabetic control, together, increased the patient's immunodeficiency, finally resulting in pulmonary infection with nocardia. The most probable route of infection is indeed a point of debate- we speculate that apart from the possibility of inhalation, the patient's history of chest trauma should be taken into consideration as well, since pulmonary nocardiosis can potentially result from exposure to bacteria via abraded skin [12].

A literature search based on PubMed/MEDLINE was conducted until July 10, 2020. A total of nine accessible cases reporting pemphigus patients with nocardiosis were retrieved (Table 1) [13-18]. One of the studies reported four cases of pulmonary nocardiosis but the presented clinical data was insufficient. The overall gender 


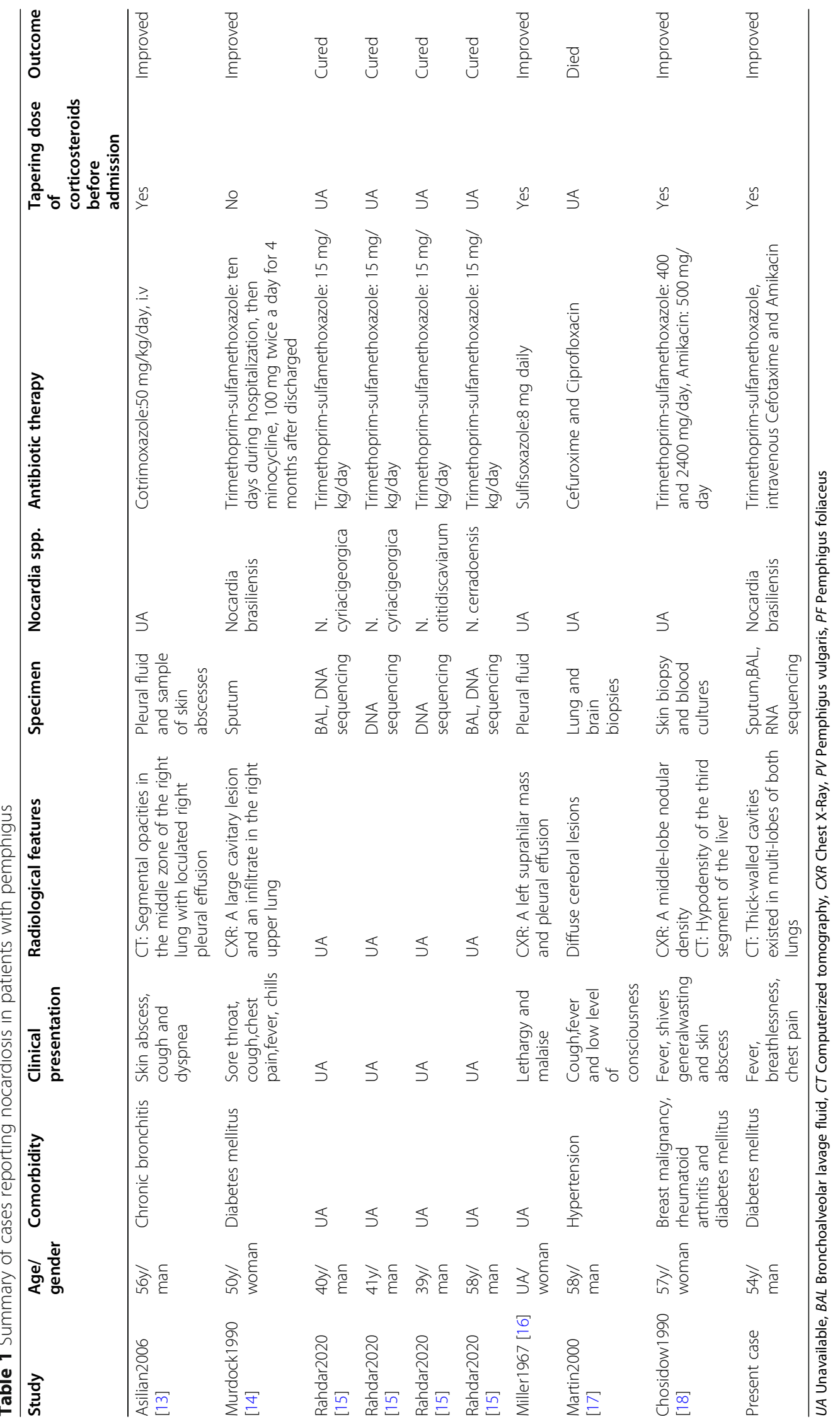


composition of studies was 3 females / 6 males. All cases were PV patients who had undergone corticosteroid treatment. Varying doses of sulfonamide antibiotics, particularly trimethoprim-sulfamethoxazole, was the most used therapeutic regimen. One patient with severely deteriorated health died within a short period of time after biopsy confirmation of nocardiosis, others improved or got cured of pulmonary nocardiosis after therapy. We noticed that except for the study with insufficient data, patients in most case-reports (4/5) had a history of tapered dose of corticosteroids in pemphigus management before presenting with and being diagnosed with nocardiosis. This brings us to a very concerning thought: was it possible that the reduced dosage of corticosteroids during the management of pemphigus resulted in immunological modifications and subsequently increased the risk of contracting opportunistic infections such as nocardiosis? If so, caution should be exercised while deciding optimal dosage as well as the time period for corticosteroid adjustment. Apart from the single patient who died right after diagnosis of nocardia, all others had a good treatment outcome. Therefore, early diagnosis along with timely and effective therapy is extremely important for the management of nocardiosis.

According to our extensive searches, this is the first case to be reported of pulmonary nocardiosis in a patient with concomitant PF. Combining the findings of the patient in this case-report and other related reports, we recommend that physicians be aware of nocardiosis in patients with pemphigus as a possible cause of underlying infectious disease to avoid misdiagnoses and mismanagement.

\section{Abbreviations}

PF: Pemphigus foliaceus; FS: Fogo selvage; PV: Pemphigus vulgaris; Dsg1: Anti-desmoglein-1; Dsg-3: Anti-desmoglein-3; UA: Unavailable; BAL: Bronchoalveolar lavage fluid; CT: Computerized tomography; CXR: Chest X-Ray

\section{Acknowledgements}

Not applicable.

\section{Authors' contributions}

N.L, SF.T,S.L,XC.L and WY. Y treated the patient and collected clinical data; This manuscript was initially drafted by S.S,MF.C,and YH. H, then revised by M. L and CN.C. CN. C provided supplementary data of the patient during the stage of revision. The authors approved the final manuscript.

\section{Funding}

This study was funded by the High-level Hospital Construction Research Project of Maoming People's Hospital, Maoming, China. The funder had no role in study design, data collection and analysis, decision to publish, or preparation of the manuscript.

\section{Availability of data and materials}

The datasets used and/or analysed during the current study are available from the corresponding author on request.

\section{Ethics approval and consent to participate}

This case report has been approved by the ethics committee of Maoming People's Hospital.

\section{Consent for publication}

Written informed consent was obtained from the patient for publication of information and images in this case-report. A copy of the written consent is available for review by the Editor of this journal.

\section{Competing interests}

The authors declare that they have no competing interests.

\section{Author details}

'Department of Respiratory and Critical Care Medicine, Maoming People's Hospital, MaoNanQu, WeiMing Road 101 Hao, Maoming City, Guangdong, China. ${ }^{2}$ Southern Medical University, Guangzhou, China. ${ }^{3}$ Department of Otorhinolaryngology, Maoming People's Hospital, Maoming, China.

${ }^{4}$ Department of Laboratory Medicine, Maoming People's Hospital, Maoming, China. ${ }^{5}$ Department of Scientific Research, Maoming People's Hospital, Maoming, China. ${ }^{6}$ Department of Dermatology, Maoming People's Hospital, Maoming, China.

Received: 1 September 2020 Accepted: 30 November 2020

Published online: 06 January 2021

\section{References}

1. Murrell DF, Peña S, Joly P, Marinovic B, Hashimoto T, Diaz LA, et al. Diagnosis and management of pemphigus: recommendations of an international panel of experts. J Am Acad Dermatol. 2020;82(3):575-85.e1.

2. Bystryn JC, Rudolph JL. Pemphigus. Lancet. 2005;366(9479):61-73.

3. Diaz LA, Sampaio SA, Rivitti EA, Martins CR, Cunha PR, Lombardi C, et al. Endemic pemphigus foliaceus (fogo selvagem). I. Clinical features and immunopathology. J Am Acad Dermatol. 1989;20(4):657-69.

4. Aoki V, Rivitti EA, Diaz LA. Update on fogo selvagem, an endemic form of pemphigus foliaceus. J Dermatol. 2015;42(1):18-26.

5. Robinson ND, Hashimoto T, Amagai M, Chan LS. The new pemphigus variants. J Am Acad Dermatol. 1999:40(5 Pt 1):649-71 quiz 72-3.

6. Alpsoy E, Akman-Karakas A, Uzun S. Geographic variations in epidemiology of two autoimmune bullous diseases: pemphigus and bullous pemphigoid. Arch Dermatol Res. 2015;307(4):291-8.

7. Carson PJ, Hameed A, Ahmed AR. Influence of treatment on the clinical course of pemphigus vulgaris. J Am Acad Dermatol. 1996;34(4):645-52.

8. Lederman ER, Crum NF. A case series and focused review of nocardiosis: clinical and microbiologic aspects. Medicine (Baltimore). 2004;83(5):300-13.

9. Tilak R, Agarwal D, Lahiri TK, Tilak V. Pulmonary nocardiosis presenting as fungal ball--a rare entity. J Infect Dev Ctries. 2008;2(2):143-5.

10. Safdar N, Kaul DR, Saint S. Clinical problem-solving. Into the woods. N Engl J Med. 2007:356(9):943-7.

11. Fatahi-Bafghi M. Nocardiosis from 1888 to 2017. Microb Pathog. 2018;114: 369-84.

12. Wilson JW. Nocardiosis: updates and clinical overview. Mayo Clin Proc. 2012; 87(4):403-7.

13. Asilian A, Yoosefi A, Faghihi G. Cutaneous and pulmonary nocardiosis in pemphigus vulgaris: a rare complication of immunosuppressive therapy. Int J Dermatol. 2006;45(10):1204-6.

14. Murdock DK, Lookingbill DP. Immunosuppressive therapy of pemphigus vulgaris complicated by Nocardia pneumonia. Gold as an alternate therapy. Arch Dermatol. 1990;126(1):27-8.

15. Rahdar HA, Bahador A, Shahraki-Zahedani S, Karami-Zarandi M, Soori T, Jafari S, Feizabadi MM. Pulmonary nocardiosis in pemphigus vulgaris patients from Tehran, Iran. Infect Disord Drug Targets. 2019. https://doi.org/10.2174/ 1871526520666191231144607. Epub ahead of print. PMID: 31889503.

16. Miller RC, Felman YM. Pemphigus vulgaris and pulmonary nocardiosis. Arch Dermatol. 1967;96(5):548-51.

17. Martín FJ, Pérez-Bernal AM, Camacho F. Pemphigus vulgaris and disseminated nocardiosis. J Eur Acad Dermatol Venereol. 2000;14(5):416-8.

18. Chosidow O, Wolkenstein P, Bagot M, Girard-Pipau F, Brun-Buisson C, Fraitag $S$, et al. Nocardia asteroides septicemia in a pemphigus patient. Successful treatment with trimethoprim-sulfamethoxazole and amikacin association. Dermatologica. 1990;181(4):311-3.

\section{Publisher's Note}

Springer Nature remains neutral with regard to jurisdictional claims in published maps and institutional affiliations. 